CLINICAL REPORT

\author{
G.N. Simao \\ S. Zarei Mahmoodabadi \\ O.C. Snead \\ C. Go \\ E. Widjaja
}

\section{Abnormal Axial Diffusivity in the Deep Gray Nuclei and Dorsal Brain Stem in Infantile Spasm Treated with Vigabatrin}

\begin{abstract}
SUMmARY: We evaluated the DTI changes in the deep gray nuclei and dorsal brain stem, which demonstrated abnormal T2 and/or diffusion signal intensity, in 6 patients with infantile spasm treated with vigabatrin compared with 6 age-matched controls. Regions of interest were placed in the globi pallidi, thalami, and dorsal brain stem; $F A$, trace, $D_{\|}$, and $D_{\perp}$ were measured. Patients on vigabatrin had significantly lower FA in both globi pallidi $(P=.01)$ and the dorsal brain stem $(P<.01)$, significantly lower trace in both globi pallidi $(P=.01)$ and the thalami $(P=.02$ and .01 for right and left, respectively), and significantly lower $\mathrm{D}_{\|}$in both globi pallidi $(P \leq .01)$, the thalami $(P<.01)$, and the dorsal brain stem $(P=.03)$. There were no significant differences in $D_{\perp}$ of the globi pallidi, thalami, or dorsal brain stem in patients compared with controls. The findings suggest that axonal changes play a greater role in the observed abnormal signal intensity, with lesser contribution from myelin changes.
\end{abstract}

V igabatrin is a structural analog of GABA, which irreversibly inhibits GABA-transaminase, increasing brain levels of GABA. Vigabatrin has been used to treat infantile spasms and refractory complex partial seizures, and clinical trials have shown that vigabatrin may provide rapid reduction in seizure frequency and has allowed some patients to become seizurefree. ${ }^{1}$ Vigabatrin was generally reported as a well-tolerated drug; however, visual disturbances, manifested as visual blurring and constriction of visual fields, were noted in some patients treated with vigabatrin. In addition, animal studies have raised concerns that prolonged administration of vigabatrin induced intramyelin edema. ${ }^{2-4}$ Recently, human studies have described reversible MR imaging abnormalities characterized by $\mathrm{T} 2$-weighted hyperintensities and/or restricted diffusion in the globi pallidi, thalami, dorsal pons, and midbrain in approximately $20 \%-30 \%$ of patients with infantile spasm. ${ }^{5-7}$ Young age and high dose appeared to be risk factors for the development of these reversible changes in the deep gray nuclei and brain stem. ${ }^{5-7}$ The underlying microstructural changes responsible for the $\mathrm{T} 2$ and diffusion changes in the human brain are unknown. However, on the basis of animal studies, we postulated that the abnormal T2 signal intensity and diffusion restriction in the deep gray nuclei and dorsal brain stem were related to intramyelin edema.

DTI can be used to indirectly evaluate the integrity of the axonal microenvironment by assessing the diffusion of water molecules and its directionality in a $3 \mathrm{D}$ space. From DTI, 3 eigenvalues $\left(\lambda_{1}, \lambda_{2}\right.$, and $\left.\lambda_{3}\right)$ are calculated. $\lambda_{1}$ or $\mathrm{D}_{\|}$represents water diffusivity parallel to the axonal fibers. The mean of $\lambda_{2}$

Received April 6, 2010; accepted after revision May 23.

From the Department of Diagnostic Imaging (G.N.S., S.Z.M., E.W.) and Division of Neurology (O.C.S., C.G., E.W.), Hospital for Sick Children, Toronto, Ontario, Canada.

Please address correspondence to Elysa Widjaja, MD, Diagnostic Imaging, Hospital for Sick Children, Toronto, Ontario M5G 1X8, Canada; e-mail: Elysa.Widjaja@sickkids.ca

DOI 10.3174/ajnr.A2224 and $\lambda_{3}$, also known as $\mathrm{D}_{\perp}$, represents water diffusion perpendicular to the axonal fibers. Animal models have shown that tensor eigenvalues were more specific markers of myelination and axonal morphology. ${ }^{8}$ Changes in $\mathrm{D}_{\|}$reflect axonal integrity and have been observed in cases of wallerian degeneration. ${ }^{9}$ In contrast, $\mathrm{D}_{\perp}$ reflects alteration in myelination. ${ }^{8,10} \mathrm{We}$ hypothesized that because the $\mathrm{T} 2$ and/or diffusion signal intensity changes in the deep gray nuclei and dorsal brain stem secondary to vigabatrin were related to intramyelin edema, DTI would demonstrate elevated $\mathrm{D}_{\perp}$ and no significant change in $\mathrm{D}_{\|}$, in addition to reduced $\mathrm{FA}$ and reduced trace. The aim of this study was to evaluate the DTI changes in the globi pallidi, thalami, and dorsal brain stem of patients with infantile spasm who had T2 and/or diffusion signal-intensity changes related to vigabatrin.

\section{Materials and Methods}

\section{Subjects}

This retrospective study had the approval of our institutional research ethics board. Six patients ( 5 males and 1 female; mean age, 9 months; age range, $7-13$ months) who underwent treatment with vigabatrin for infantile spasm and developed abnormal signal intensity in the globi pallidi, thalami, and/or dorsal brain stem on T2-weighted imaging and/or diffusion-weighted imaging were recruited for the study. Six age- and sex-matched control subjects ( 5 males and 1 female; mean age, 1.0 year; age range, $0.6-1.5$ years) with no history of neurologic disorders who were imaged due to cutaneous lesions and had normal findings on MR imaging and DTI were included in the study. All 6 patients underwent DTI in addition to structural MR and diffusion-weighted imaging. The etiology of infantile spasm, dose of vigabatrin, and duration of vigabatrin treatment at the time of imaging are presented in Table 1. None of the patients had hypoxic injury or metabolic diseases that could explain the abnormal signal intensity in the globi pallidi, thalami, and/or dorsal brain stem. 


\begin{tabular}{|c|c|c|c|c|c|c|c|c|}
\hline \multirow{2}{*}{$\begin{array}{l}\text { Patient } \\
\text { No. }\end{array}$} & \multirow[b]{2}{*}{ Sex } & \multirow[b]{2}{*}{ Diagnosis } & \multirow{2}{*}{$\begin{array}{c}\text { Age at } \\
\text { MRI (mo) }\end{array}$} & \multirow{2}{*}{$\begin{array}{c}\text { Duration of } \\
\text { VGB at MRI } \\
\text { (days) }\end{array}$} & \multirow{2}{*}{$\begin{array}{c}\text { Dose (mg) } \\
\text { of VGB at } \\
\text { time of MRI }\end{array}$} & \multicolumn{3}{|c|}{$\begin{array}{c}\text { Areas of T2 and/or Diffusion } \\
\text { Hyperintensity }\end{array}$} \\
\hline & & & & & & GP & Thalamus & $\mathrm{DB}$ \\
\hline 1 & $M$ & Cryptogenic IS & 8 & 49 & 1250 & Yes & No & Yes \\
\hline 2 & $\mathrm{~F}$ & Cryptogenic IS & 7 & 32 & 1400 & Yes & No & No \\
\hline 3 & $\mathrm{M}$ & Cryptogenic IS & 8 & 67 & 1500 & Yes & Yes & Yes \\
\hline 4 & $\mathrm{M}$ & TSC & 13 & 230 & 1375 & Yes & No & No \\
\hline 5 & $\mathrm{M}$ & Cryptogenic IS & 13 & 17 & 1630 & Yes & No & No \\
\hline 6 & $\mathrm{M}$ & Cryptogenic IS & 9 & 52 & 1250 & Yes & Yes & Yes \\
\hline
\end{tabular}

\section{MR Imaging and DTI}

MR imaging was performed with a 3T magnet (Achieva; Phillips Healthcare, Best, the Netherlands) and an 8-channel head coil. Imaging protocol consisted of a variety of sequences, including sagittal 3D T1-weighted sequence to cover the entire brain (TR/TE, 4.9/2.3 ms; section thickness, $1 \mathrm{~mm}$; FOV, $22 \mathrm{~cm}$; matrix, $220 \times 220$ ), axial and coronal T2-weighted images (TR/TE, 4200/80 ms; section thickness, 3 $\mathrm{mm}$; FOV, $22 \mathrm{~cm}$; matrix, $400 \times 272$ ), axial fluid-attenuated inversion recovery images (TR/TE, 10,000/140 ms; section thickness, 3 $\mathrm{mm}$; FOV, $22 \mathrm{~cm}$; matrix, $316 \times 290$ ), and axial diffusion-weighted images (TR/TE, 2200/50 ms; section thickness, $5 \mathrm{~mm}$; FOV, $22 \mathrm{~cm}$; matrix, $120 \times 118)$.

Axial DTI was performed on the same MR imaging scanner in both patients and controls, by using single-shot diffusion-weighted echo-planar imaging: 15 directions; TR/TE, 11000/55 ms; section thickness, $2 \mathrm{~mm}$; FOV, $22 \mathrm{~cm}$; matrix, $112 \times 112$; b-value, 1000 $\mathrm{s} / \mathrm{mm}^{2}$ and $0 \mathrm{~s} / \mathrm{mm}^{2}$; and NEX, 2. Eddy current correction was done automatically on the scanner.

\section{Data Analysis}

DTI data processing was performed with DTIStudio, Version 2.4 (Johns Hopkins University, Baltimore, Maryland; https://www.mristudio.org/). Diagonalization of the diffusion tensor yielded 6 independent tensor elements: 3 eigenvalues $\left(\lambda_{1}, \lambda_{2}\right.$, and $\left.\lambda_{3}\right)$ and 3 eigenvectors, which provided $3 \mathrm{D}$ information about the diffusivity of water molecules per voxel that can be represented graphically by an ellipsoid. ${ }^{11}$ From these data, the FA, trace, and eigenvalue $\left(\lambda_{1}, \lambda_{2}\right.$, and $\left.\lambda_{3}\right)$ maps were calculated. Regions of interest were carefully drawn by 2 neuroradiologists (G.N.S. and E.W.) to outline the globi pallidi, thalami, and dorsal brain stem on an FA map (Fig 1). The same regions of interest were then transposed onto trace and eigenvalues maps. FA, trace, $\mathrm{D}_{\|}$, and $\mathrm{D}_{\perp}$ were evaluated.

\section{Statistical Analysis}

Data were analyzed by using the Statistical Package for the Social Sciences, Version 15 (SPSS, Chicago, Illinois). The inter-rater agreement on $\mathrm{FA}$, trace, $\mathrm{D}_{\|}$, and $\mathrm{D}_{\perp}$ of the right and left globi pallidi, thalami, and dorsal brain stem were evaluated by using the intraclass correlation coefficient. An intraclass correlation coefficient of $0-0.2$ indicated poor agreement; 0.21-0.40, fair agreement; 0.41-0.60, moderate agreement; $0.61-0.80$, substantial agreement; and $0.81-1.0$, nearly perfect agreement. The mean DTI indices of the 2 assessors were used for subsequent analysis. A nonparametric Mann-Whitney $U$ test was used to compare $\mathrm{FA}$, trace, $\mathrm{D}_{\|}$, and $\mathrm{D}_{\perp}$ of the globi pallidi, thalami, and dorsal brain stem of the patients versus controls. A nonparametric Mann-Whitney $U$ test was also used to compare FA, trace, $\mathrm{D}_{\|}$, and $\mathrm{D}_{\perp}$ of the right and left thalami and the dorsal brain stem of patients with abnormal T2 and/or diffusion signal intensity with those with normal T2 and diffusion signal intensity. Statistical significance was set at $P<.05$.

\section{Results}

There was near-perfect agreement between the 2 raters in the DTI indices of the right globus pallidus (ICC of 0.91 to 0.99 ), left globus pallidus (ICC of 0.87 to 0.97 ), right thalamus (ICC of 0.94 to 0.99 ), and left thalamus (ICC of 0.98 to 0.99 ) of patients and controls. There was substantial-to-near-perfect agreement of the DTI indices in the dorsal brain stem (ICC of 0.75 to 0.97 ) of patients and controls.

Patients on vigabatrin therapy who had abnormal T2 and/or diffusion signal-intensity findings on MR imaging had significantly lower FA in the right (mean $\mathrm{FA}=0.19, P=.01$ ) and left (mean FA $=0.21, P=.01$ ) globi pallidi and dorsal brain stem (mean FA $=0.49, P<.01$ ) compared with controls (mean FA of the right and left globi pallidi were 0.25 and 0.26 , respectively, and mean FA of the dorsal brain stem was 0.60 ; Table 2). Trace was significantly lower in the right (mean trace $=2.23, P=.01)$ and left (mean trace $=2.18, P=.01)$ globi pallidi and right (mean trace $=2.14, P=.02$ ) and left (mean trace $=2.17, P=.01$ ) thalami in patients compared with controls (mean trace of the right and left globi pallidi was 2.14 and 2.17, respectively, and mean trace of the right and left thalami was 2.53 and 2.51 , respectively). $\mathrm{D}_{\|}$was significantly lower in the right (mean $\mathrm{D}_{\|}=0.88, P=.01$ ) and left (mean $\mathrm{D}_{\|}$ $=0.87, P<.01)$ globi pallidi, right $\left(\right.$ mean $\left.\mathrm{D}_{\|}=0.89, P<.01\right)$ and left (mean $\mathrm{D}_{\|}=0.89, P<.01$ ) thalami, and the dorsal brain stem (mean $\mathrm{D}_{\|}=1.13, P=.03$ ) in patients compared with controls (means of $\mathrm{D}_{\|}$of the right and left globi pallidi were 1.12 and 1.15 , respectively; the mean $\mathrm{D}_{\|}$of the right and left thalami was 1.04 bilaterally; and the mean $\mathrm{D}_{\|}$of the dorsal brain stem was 1.41). There were no significant differences in $\mathrm{D}_{\perp}$ in the globi pallidi, thalami, and dorsal brain stem of patients compared with controls.

All 6 patients had abnormal T2 and/or diffusion signal intensity in the globi pallidi, 2 had abnormal T2 and/or diffusion signal intensity in the thalami, and $3 \mathrm{had}$ abnormal T2 and/or diffusion signal intensity in the dorsal brain stem. There were no significant differences in FA, trace, $\mathrm{D}_{\|}$, and $\mathrm{D}_{\perp}$ of those with abnormal T2 and/or diffusion signal intensity compared with those with normal T2 and diffusion signal intensity in the thalami and dorsal brain stem $(P \geq .05)$. One patient with normal T2 and diffusion signal intensity in the right and left thalami had decreased trace, $\mathrm{D}_{\|}$, and $\mathrm{D}_{\perp}$, which were 2 SDs below the mean values of controls. Another patient with normal T2 and diffusion signal intensity in the right and left thalami had reduced FA, trace, $\mathrm{D}_{\|}$, and $\mathrm{D}_{\perp}$, which were 2 SDs 

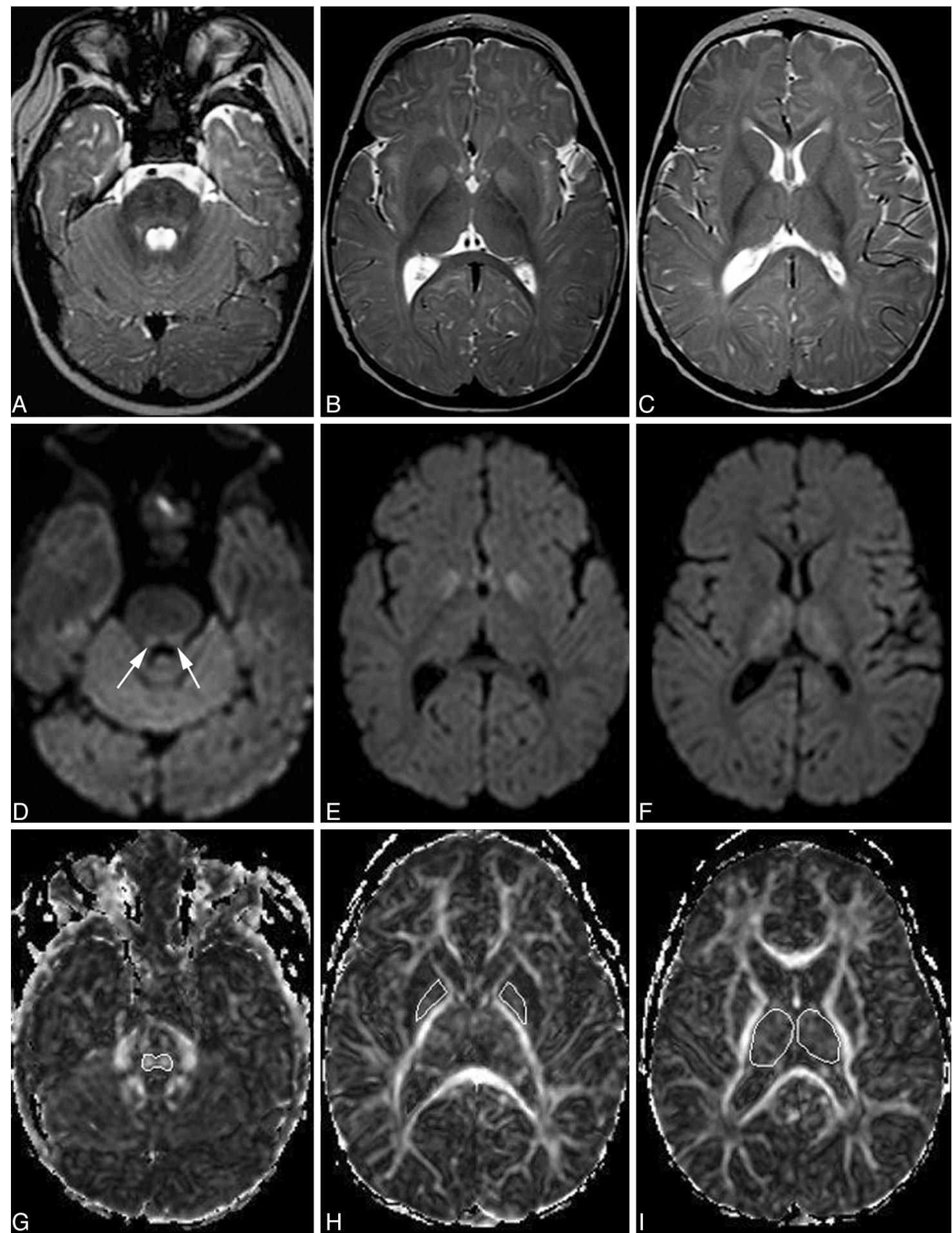

Fig 1. A 9-month-old with infantile spasm treated with vigabatrin. $A-F$, Axial T2 $(A-C)$ and diffusion-weighted $(D-F)$ images show increased T2 signal intensity in the globi pallidi; subtle increased T2 signal intensity in the thalami and dorsal brain stem; and increased diffusion signal intensity in the dorsal brain stem (arrows), globi pallidi, and thalami. G-I, Regions of interest are carefully drawn over the FA maps in the globi pallidi $(G)$, thalami $(H)$, and dorsal brain stem $(\Lambda$.

below the mean values of controls. One patient with normal $\mathrm{T} 2$ and diffusion signal intensity in the dorsal brain stem had reduced $F A$ and $D_{\|}$, which were $2 S D$ s below the mean values of controls.

\section{Discussion}

We found significantly reduced trace and significantly reduced $\mathrm{D}_{\|}$in the globi pallidi and thalami, but no significant elevation in $\mathrm{D}_{\perp}$. Animal studies suggested that a reduction in $\mathrm{D}_{\|}$could be related to an abnormality in the axonal structure rather than abnormal myelin. Beaulieu et al ${ }^{12}$ studied wallerian degeneration of the frog sciatic nerve and observed that most axons had either collapsed or were degraded and permeated with amorphous granular material instead of containing normal neurofilaments and mitochondria. The authors attributed the reduction in $\mathrm{D}_{\|}$to breakdown of the longitudinal 


\begin{tabular}{|c|c|c|c|c|c|c|c|c|c|c|c|c|c|}
\hline & & \multicolumn{3}{|c|}{ Mean Trace $\times 10^{-3}(\mathrm{SD})$} & \multicolumn{3}{|c|}{ Mean FA (SD) } & \multicolumn{3}{|c|}{ Mean $\mathrm{D}_{\|} \times 10^{-3}(\mathrm{SD})$} & \multicolumn{3}{|c|}{ Mean $D_{\perp} \times 10^{-3}(S D)$} \\
\hline & & & & $P$ & & & $P$ & & & $P$ & & & $P$ \\
\hline & & VGB-Pt & Controls & Value & VGB-Pt & Controls & Value & VGB-Pt & Controls & Value & VGB-Pt & Controls & Value \\
\hline \multirow[t]{2}{*}{$\overline{\mathrm{GP}}$} & $\mathrm{R}$ & $2.23(.41)$ & $2.66(.13)$ & .01 & $0.19(.04)$ & $0.25(.02)$ & $<.01$ & $0.88(.18)$ & $1.12(.06)$ & .01 & $0.67(.12)$ & $0.77(.04)$ & .10 \\
\hline & $\mathrm{L}$ & $2.18(.49)$ & $2.70(.13)$ & .01 & $0.21(.03)$ & $0.26(.03)$ & .01 & $0.87(.20)$ & $1.15(.07)$ & $<.01$ & $0.65(.14)$ & $0.77(.04)$ & .07 \\
\hline \multirow[t]{2}{*}{ Thal } & $\mathrm{R}$ & $2.14(.56)$ & $2.53(.06)$ & .02 & $0.23(.03)$ & $0.24(.02)$ & .44 & $0.89(.23)$ & $1.04(.03)$ & $<.01$ & $0.63(.16)$ & $0.74(.02)$ & .05 \\
\hline & $\mathrm{L}$ & $2.17(.54)$ & $2.51(.06)$ & .01 & $0.23(.04)$ & $0.25(.02)$ & .10 & $0.89(.22)$ & $1.04(.02)$ & $<.01$ & $0.65(.15)$ & $0.74(.02)$ & .18 \\
\hline \multicolumn{2}{|l|}{ DB } & $2.13(.40)$ & $2.37(.19)$ & .29 & $0.49(.09)$ & $0.6(.04)$ & $<.01$ & $1.13(.26)$ & $1.41(.15)$ & .03 & $0.5(.09)$ & $0.49(.04)$ & .84 \\
\hline
\end{tabular}

axonal structure. They also observed an increase in $\mathrm{D}_{\perp}$, which was attributed to breakdown of myelin by macrophages, hence loss of the distinction of intra- and extra-axonal water environments. Song et $\mathrm{al}^{8}$ examined the optic nerve serially by using DTI following ischemia and observed a reduction in $\mathrm{D}_{\|} 3$ days after ischemia without concurrent detectable changes in $\mathrm{D}_{\perp}$. The reduction in $\mathrm{D}_{\|}$correlated with histologic findings of axonal degeneration with no detectable evidence of demyelination. The elevation of $D_{\perp}$ was observed 5 days after ischemia, and this correlated with histologic findings of myelin degeneration.

Our findings of significantly reduced $\mathrm{D}_{\|}$suggested that derangement in axonal structure might play a dominant role in the observed DTI changes in the deep gray nuclei and dorsal brain stem. This is contrary to the findings described in animals that have been exposed to vigabatrin. A randomized placebo-control study of 108 dogs given vigabatrin for $\leq 12$ weeks demonstrated myelin vacuolation in the thalamus, hypothalamus, and fornix on histology $4-5$ weeks after treatment. ${ }^{4}$ The ages of the dogs were not stated. MR imaging changes were present in these animals several weeks after histologic changes. There was increased T2 signal intensity in the hypothalamus 4 weeks after treatment and in the thalamus and fornix 7 weeks after treatment. The MR imaging signal intensity increased with the duration of the treatment and normalized 16 weeks after the drug was discontinued. ${ }^{4}$

Myelin vacuolation has been reported in the myelinated white matter tracts of mice exposed to vigabatrin. ${ }^{2}$ Further study of rats that have been treated with vigabatrin showed lesions in the cerebellar white matter and increased T2 relaxation on MR imaging, which correlated with microvacuolation on postmortem examination. ${ }^{3}$ At the time of vigabatrin administration, these rats were 4 weeks of age and myelination was expected to have been completed at that point in time. Increased T2 relaxation on MR imaging was also seen in the cerebral cortex, thalamus, and hippocampus, which correlated with reactive astrocytes and, in more severe cases, microglial proliferation and microvacuoles. ${ }^{3}$

Besides myelin microvacuolation, animal studies have also found other histologic abnormalities in immature animals exposed to vigabatrin. Sidhu et $\mathrm{al}^{13}$ examined immature rats from 12 to 16 days of age exposed to clinically comparable doses of vigabatrin $(15-50 \mathrm{mg} / \mathrm{kg} /$ day $)$ and demonstrated axonal degeneration and glial cell death in the white matter and reactive astrogliosis in the frontal cortex. However, they did not detect myelin vacuolation. Qiao et $\mathrm{al}^{14}$ studied the longterm effects of vigabatrin administration on the immature rat brain. At the end of 2 weeks of vigabatrin administration, they found abnormal signal intensity on T2 and diffusion-weighted imaging and observed microvacuolation, dying cells, and swollen axons in the corpus callosum. Two weeks after cessation of vigabatrin administration, there was no abnormal signal intensity on MR imaging, but the T2 relaxation time remained elevated in the hypothalamus and the reduction of the myelination reversed partially. ${ }^{14}$ In explants of rat cerebellum exposed to vigabatrin, there was no evidence of intramyelin edema or primary demyelination, but degenerating myelinated fibers and astrocytic gliosis were seen on electron microscopy. ${ }^{15}$ It is possible that axonal swelling, axonal degeneration, reactive astrogliosis, or an as-yet-unknown factor may have, in part, contributed to the significantly reduced $D_{\|}$in the deep gray nuclei and dorsal brain stem in our patients.

Immature animals were more likely to demonstrate histologic findings other than myelin vacuolation, such as axonal swelling, axonal degeneration, and astrocytic gliosis ${ }^{15}$ compared with myelin-mature animals. Previous studies have shown that children younger than 2 years of age were more likely to demonstrate abnormal signal intensity in the deep gray nuclei and dorsal brain stem compared with older children or adults when exposed to vigabatrin..$^{5,7,16}$ The observed abnormal signal intensity in the deep gray nuclei and dorsal brain stem in infants with relatively immature myelin exposed to vigabatrin may not be related to myelin vacuolationhence, the lack of significant alteration of $\mathrm{D}_{\perp}$ in the globi pallidi, thalami, and dorsal brain stem in our cohort of patients.

In studies involving animal models, the question often remains as to whether the findings are generalizable to humans. To date, there has been 1 postmortem case of a child with a history of hypoxic injury due to prematurity who received vigabatrin at 9 months of age for the treatment of infantile spasm. ${ }^{17}$ This child underwent vigabatrin treatment for 3 days, and 9 days later underwent vigabatrin treatment for a total of 11 days before dying of bronchopneumonia. Postmortem findings demonstrated microglial activation in the white matter and the CA1 sector of the hippocampus as well as the presence of large vacuoles within the myelin in the brain stem and white matter associated with the globi pallidi, dentate nucleus, and white matter of some cerebellar folia. There was no evidence of reactive astrocytes, reactive microglia, or damaged axons associated with the vacuoles. It is not known whether both myelin vacuolation and axonal changes could occur in infants exposed to vigabatrin or whether myelin vacuolation represents early changes and axonal changes developed later. The observed reduced $D_{\|}$in this study may be related to the longer exposure to vigabatrin because the mean duration of vigabatrin exposure in our patients was 74.5 days. Further longitudinal studies are needed to determine if DTI indices of $\mathrm{D}_{\|}$ and $\mathrm{D}_{\perp}$ alter during the course of vigabatrin treatment. 


\section{Conclusions}

We have found significantly reduced FA, trace, and $\mathrm{D}_{\|}$and no significant alteration in $\mathrm{D}_{\perp}$ in the deep gray nuclei and dorsal brain stem of infants with $\mathrm{T} 2$ and/or diffusion changes related to vigabatrin treatment for infantile spasm. Significant reduction in $\mathrm{D}_{\|}$suggested that axonal abnormalities might have contributed to the observed MR imaging signal-intensity changes. We have not found any significant alteration in $\mathrm{D}_{\perp}$ in our cohort of patients, suggesting that changes in myelin may have a lesser role in the observed signal-intensity changes. Alternatively, failure to detect a significant change in $\mathrm{D}_{\perp}$ could be due to the relatively small sample size in our study. More human studies are necessary to validate our observations.

\section{References}

1. Willmore LJ, Abelson MB, Ben-Menachem E, et al. Vigabatrin: 2008 update. Epilepsia 2009;50:163-73

2. Butler WH, Ford GP, Newberne JW. A study of the effects of vigabatrin on the central nervous system and retina of Sprague Dawley and Lister-Hooded rats. Toxicol Pathol 1987;15:143-48

3. Jackson GD, Williams SR, Weller RO, et al. Vigabatrin-induced lesions in the rat brain demonstrated by quantitative magnetic resonance imaging. Epilepsy Res 1994;18:57-66

4. Peyster RG, Sussman NM, Hershey BL, et al. Use of ex vivo magnetic resonance imaging to detect onset of vigabatrin-induced intramyelinic edema in canine brain. Epilepsia 1995;36:93-100

5. Dracopoulos A, Widjaja E, Raybaud C, et al. Vigabatrin-associated reversible MRI signal changes in patients with infantile spasms. Epilepsia 2010;51:1297-304
6. Milh M, Villeneuve N, Chapon F, et al. Transient brain magnetic resonance imaging hyperintensity in basal ganglia and brain stem of epileptic infants treated with vigabatrin. $J$ Child Neurol 2009;24:305-15

7. Pearl PL, Vezina LG, Saneto RP, et al. Cerebral MRI abnormalities associated with vigabatrin therapy. Epilepsia 2009;50:184-94

8. Song SK, Sun SW, Ju WK, et al. Diffusion tensor imaging detects and differentiates axon and myelin degeneration in mouse optic nerve after retinal ischemia. Neuroimage 2003;20:1714-22

9. Thomalla G, Glauche V, Koch MA, et al. Diffusion tensor imaging detects early wallerian degeneration of the pyramidal tract after ischemic stroke. Neuroimage 2004;22:1767-74

10. Song SK, Sun SW, Ramsbottom MJ, et al. Dysmyelination revealed through MRI as increased radial (but unchanged axial) diffusion of water. Neuroimage 2002;17:1429-36

11. Basser PJ, Pierpaoli C. Microstructural and physiological features of tissues elucidated by quantitative-diffusion-tensor MRI. J Magn Reson B 1996;111:209-19

12. Beaulieu C, Does MD, Snyder RE, et al. Changes in water diffusion due to wallerian degeneration in peripheral nerve. Magn Reson Med 1996;36:627-31

13. Sidhu RS, Del Bigio MR, Tuor UI, et al. Low-dose vigabatrin (gamma-vinyl GABA)-induced damage in the immature rat brain. Exp Neurol 1997;144:400-05

14. Qiao M, Malisza KL, Del Bigio MR, et al. Effect of long-term vigabatrin administration on the immature rat brain. Epilepsia 2000;41:655-65

15. Hauw JJ, Boutry JM, Sun P, et al. Effects of vigabatrin and of GABA on myelinated rat cerebellar cultures: preliminary data. Br J Clin Pharmacol 1989; 27(suppl 1):47S-52S

16. Wheless JW, Carmant L, Bebin M, et al. Magnetic resonance imaging abnormalities associated with vigabatrin in patients with epilepsy. Epilepsia 2009;50:195-205

17. Horton M, Rafay M, Del Bigio MR. Pathological evidence of vacuolar myelinopathy in a child following vigabatrin administration. J Child Neurol 2009; 24:1543-46 